\title{
The Hamiltonian problem and $t$-path traceable graphs
}

\author{
Kashif Bari \\ Department of Mathematics and Statistics \\ San Diego State University \\ San Diego, California \\ kashbari@math.tamu.edu
}

\author{
Michael E. O'Sullivan \\ Department of Mathematics and Statistics \\ San Diego State University \\ San Diego, California \\ mosullivan@mail.sdsu.edu
}

\begin{abstract}
The problem of characterizing maximal non-Hamiltonian graphs may be naturally extended to characterizing graphs that are maximal with respect to non-traceability and beyond that to $t$-path traceability. We show how traceability behaves with respect to disjoint union of graphs and the join with a complete graph. Our main result is a decomposition theorem that reduces the problem of characterizing maximal $t$-path traceable graphs to characterizing those that have no universal vertex. We generalize a construction of maximal non-traceable graphs by Zelinka to $t$-path traceable graphs.
\end{abstract}

\section{Introduction}

The motivating problem for this article is the characterization of maximal non-Hamiltonian $(\mathrm{MNH})$ graphs. Skupien and co-authors give the first broad family of MNH graphs in [6] and describe all MNH graphs with 10 or fewer vertices in 2. The latter paper also includes three constructions - types $A 1, A 2, A 3$-with a similar structure. Zelinka gave two constructions of graphs that are maximal non-traceable; that is, they have no Hamiltonian path, but the addition of any edge gives a Hamiltonian path. The join of such a graph with a single vertex gives a MNH graph. Zelinka's first family produces, under the join with $K_{1}$, the Skupien MNH graphs from [6]. Zelinka's second family is a broad generalization of the type $A 1, A 2$, and $A 3$ graphs of [2]. Bullock et al [1] provide further examples of infinite families of maximal non-traceable graphs.

In this article we work with two closely related invariants of a graph $G, \check{\mu}(G)$ and $\mu(G)$. The $\mu$-invariant, introduced by Ore [5], is the maximal number of paths in $G$ required to cover the vertex set of $G$. We show that $\check{\mu}(G)=\mu(G)$ unless $G$ is Hamiltonian, when $\check{\mu}(G)=0$. Maximal non-Hamiltonian graphs are maximal with respect to $\check{\mu}(G)=1$, and maximal non-traceable graphs are maximal with respect to $\breve{\mu}(G)=2$. It is useful to broaden the perspective to study, for arbitrary $t$, graphs that are maximal with respect to $\check{\mu}(G)=t$, which we call $t$-path traceable graphs.

In Section 2 we show how the $\check{\mu}$ and $\mu$ invariants behave with respect to disjoint union of graphs and the join with a complete graph. Section 3 derives the main result, a decomposition theorem that reduces the problem of characterizing maximal $t$-path traceable to characterizing those that have no universal vertex, which we call trim. Section 4 presents a generalization of the Zelinka construction to $t$-path traceable graphs. 


\section{Traceability and Hamiltonicity}

It will be notationally convenient to say that the complete graphs $K_{1}$ and $K_{2}$ are Hamiltonian. As justification for this view, consider an undirected graph as a directed graph with each edge having a conjugate edge in the reverse direction. This perspective does not affect the Hamiltonicity of a graph with more than 3 vertices, but it does give $K_{2}$ a Hamiltonian cycle. Similarly, adding loops to any graph with more than 2 vertices does not alter the Hamiltonicity of the graph, but $K_{1}$, with an added loop, has a Hamiltonian cycle.

Let $G$ be a graph. A vertex, $v \in V(G)$, is called a universal vertex if $\operatorname{deg}(v)=|V(G)|-1$. Let $\bar{G}$ denote the graph complement of $G$, having vertex set $V(G)$ and edge set $E\left(K_{n}\right) \backslash E(G)$. We will use the disjoint union of two graphs, $G \sqcup H$ and the join of two graphs $G * H$. The latter is $G \sqcup H$ together with the edges $\{v w \mid v \in V(G)$ and $w \in V(H)\}$.

Definition 1. A set of $s$ disjoint paths in a graph $G$ that includes every vertex in $G$ is a s-path covering of $G$. Define the following invariants.

$$
\begin{aligned}
& \mu(G):=\min _{s \in \mathbb{N}}\{\exists s \text {-path covering of } G\} . \\
& \check{\mu}(G):=\min _{l \in \mathbb{N}_{0}}\left\{K_{l} * G \text { is Hamiltonian }\right\} \\
& i_{H}(G):= \begin{cases}1 & \text { if } G \text { is Hamiltonian } \\
0 & \text { otherwise }\end{cases}
\end{aligned}
$$

We will say $G$ is $t$-path traceable when $\mu(G)=t$. A set of $t$ disjoint paths that cover a $t$-path traceable graph $G$ is a minimal path covering.

Note that $K_{r} *\left(K_{s} * G\right)=K_{r+s} * G$. If $G$ is Hamiltonian then so is $K_{r} * G$ for $r \geqslant 0$. (In particular this is true for $G=K_{1}$ and $G=K_{2}$.)

We now have a series of lemmas that lead to the main result of this section, which is a formula showing how the $\mu$-invariant and $\breve{\mu}$-invariant behave with respect to disjoint union and the join with a complete graph.

Lemma 2. $\check{\mu}(G)=\min _{l \in \mathbb{N}_{0}}\left\{\overline{K_{l}} * G\right.$ is Hamiltonian $\}$

Proof. Since $\overline{K_{l}} * G$ is a subgraph of $K_{l} * G$, a Hamiltonian cycle in $\overline{K_{l}} * G$ would also be one in $K_{l} * G$.

Let $\check{\mu}(G)=a$. Suppose $C$ is a Hamiltonian cycle in $K_{a} * G$ and write $C$ as $v \sim P_{1} \sim Q_{1} \sim$ $\ldots \sim P_{s} \sim Q_{s} \sim v$, where $v$ is a vertex in $G$ and the paths $P_{i} \in G$ and $Q_{i} \in K_{a}$. If any $Q_{i}$ contains 2 vertices or more, say $u$ and $w_{1}, \ldots, w_{k}$ with $k \geqslant 1$, then we may simply remove all the vertices, except $u$, and end up with a Hamiltonian graph on $K_{a-k}$. This contradicts the minimality of $a=\check{\mu}(G)$. Therefore, $C$ must not contain any paths of length greater than two in the subgraph $K_{a}$, and any Hamiltonian cycle on $K_{a} * G$ is also a Hamiltonian cycle on $\overline{K_{a}} * G$.

Lemma 3. $\check{\mu}(G)=\mu(G)-i_{H}(G)$

Proof. If $G$ is Hamiltonian (including $P_{1}$ and $P_{2}$ ) then $\check{\mu}(G)=0, \mu(G)=1$ so the equality holds. Suppose $G$ is non-Hamiltonian with $\mu(G)=t$ and $t$-path covering $P_{1}, \ldots, P_{t}$. Let $K_{t}$ 
have vertices $u_{1}, \ldots, u_{t}$. In the graph $K_{t} * G$, there is a Hamiltonian cycle: $v_{1} \sim P_{1} \sim v_{2} \sim P_{2} \sim$ $\cdots \sim v_{t} \sim P_{t} \sim v_{1}$. Thus $\check{\mu}(G) \leqslant t=\mu(G)$.

Let $\check{\mu}(G)=a$, so there is a Hamiltonian cycle in $K_{a} * G$. Removing the vertices of $K_{a}$ breaks the cycle into at most $a$ disjoint paths covering $G$. Thus $\mu(G) \leqslant \check{\mu}(G)$.

Lemma 4. $\mu(G \sqcup H)=\mu(G)+\mu(H)$ and $\check{\mu}(G \sqcup H)=\check{\mu}(G)+\check{\mu}(H)+i_{H}(G)+i_{H}(H)$.

Proof. A path covering of $G$ may be combined with a path covering of $H$ to create one for $G \sqcup H$. Conversely, paths in a $t$-path covering of $G \sqcup H$ can be partitioned into those contained in $G$ and those contained in $H$, giving a path covering of $G$ and one of $H$. Consequently

$$
\mu(G \sqcup H)=\mu(G)+\mu(H)
$$

Since $G \sqcup H$ is not Hamiltonian we have

$$
\begin{aligned}
\check{\mu}(G \sqcup H) & =\mu(G \sqcup H)+i_{H}(G \sqcup H) \\
& =\mu(G)+\mu(H) \\
& =\check{\mu}(G)+i_{H}(G)+\check{\mu}(H)+i_{H}(H)
\end{aligned}
$$

Lemma 5. For any graph $G$,

$$
\begin{aligned}
& \mu\left(K_{s} * G\right)=\max \{1, \mu(G)-s\} \\
& \check{\mu}\left(K_{s} * G\right)=\max \{0, \check{\mu}(G)-s\}
\end{aligned}
$$

In particular, if $K_{s} * G$ is Hamiltonian then $\mu\left(K_{s} * G\right)=1$ and $\check{\mu}\left(K_{s} * G\right)=0$; otherwise, $\mu\left(K_{s} * G\right)=\mu(G)-s$ and $\check{\mu}\left(K_{s} * G\right)=\check{\mu}(G)-s$.

Proof. The formula for $\check{\mu}$ is immediate when $G$ is Hamiltonian since we have observed that this forces $K_{s} * G$ to be Hamiltonian. Otherwise, it follows from $K_{r} *\left(K_{s} * G\right)=K_{r+s} * G$ : if $\check{\mu}(G)=a$, then $K_{r} *\left(K_{s} * G\right)$ is Hamiltonian if and only if $r+s \geqslant a$.

The formula for $\mu$ may be derived from the result for $\check{\mu}$ using Lemma 3 . We may also prove it directly. Observe that it is enough to prove $\mu\left(K_{1} * G\right)=\max \{1, \mu(G)-1\}$. Let $u$ be the vertex of $K_{1}$. Let $\mu(G)=t$ and $P_{1}, \ldots, P_{t}$ a $t$-path covering of $G$. If $t=1$ then $u$ can be connected to the initial vertex of $P_{1}$ to create a 1-path covering of $K_{1} * G$. For $t \geqslant 2$, the path $P_{1} \sim u \sim P_{2}$ along with $P_{3}, \ldots, P_{t}$ gives a $(t-1)$-path covering of $K_{1} * G$. Thus for $t>1$, $\mu\left(K_{1} * G\right) \leqslant t-1$. Suppose $Q_{1}, \ldots, Q_{d}$ were a minimal $d$-path covering of $K_{1} * G$, with $u$ a vertex of $Q_{1}$. Removing $u$ gives at most a $(d+1)$-path covering of $G$. Thus $\mu\left(K_{1} * G\right)+1 \geqslant t$. This shows $\mu\left(K_{1} * G\right)=\mu(G)-1$ for $\mu(G) \geqslant 2$.

The main result of this section is the following two formulas for for the $\mu$ and $\check{\mu}$ invariants for the disjoint union of graphs, and the join with a complete graph. 
Proposition 6. Let $\left\{G_{j}\right\}_{j=1}^{m}$ be graphs.

$\mu\left(\bigsqcup_{j=1}^{m} G_{j}\right)=\sum_{j=1}^{m} \mu\left(G_{j}\right)$ and $\check{\mu}\left(\bigsqcup_{j=1}^{m} G_{j}\right)=\sum_{j=1}^{m} \check{\mu}\left(G_{j}\right)+\sum_{j=1}^{m} i_{H}\left(G_{j}\right)$.

Furthermore, $\check{\mu}\left(\left(\bigsqcup_{j=1}^{m} G_{j}\right) * K_{r}\right)=\max \left\{0, \sum_{j=1}^{m} \check{\mu}\left(G_{j}\right)+\sum_{j=1}^{m} i_{H}\left(G_{j}\right)-r\right\}$.

Proof. We proceed by induction. The base case $k=2$ is exactly Lemma 4. Assume the formula holds for $k$ graphs we will prove it for $k+1$ graphs.

$$
\begin{aligned}
\mu\left(\bigsqcup_{j=1}^{k+1} G_{j}\right) & =\mu\left(\left(\bigsqcup_{j=1}^{k+1} G_{j}\right) \sqcup G_{k+1}\right) \\
& =\mu\left(\bigsqcup_{j=1}^{k} G_{j}\right)+\mu\left(G_{k+1}\right) \\
& =\sum_{j=1}^{k} \mu\left(G_{j}\right)+\mu\left(G_{k+1}\right) \\
& =\sum_{j=1}^{k+1} \mu\left(G_{j}\right)
\end{aligned}
$$

By Lemma 3 and the fact that disjoint graphs are not Hamiltonian, we have,

$$
\begin{aligned}
\check{\mu}\left(\bigsqcup_{j=1}^{m} G_{j}\right) & =\mu\left(\bigsqcup_{j=1}^{m} G_{j}\right)+i_{H}\left(\bigsqcup_{j=1}^{m} G_{j}\right) \\
& =\sum_{j=1}^{m} \mu\left(G_{j}\right)+0 \\
& =\sum_{j=1}^{m}\left(\check{\mu}\left(G_{j}\right)+i_{H}\left(G_{j}\right)\right) \\
& =\sum_{j=1}^{m} \check{\mu}\left(G_{j}\right)+\sum_{j=1}^{m} i_{H}\left(G_{j}\right)
\end{aligned}
$$

Therefore, we have by Lemma 5 ,

$$
\begin{aligned}
\check{\mu}\left(\left(\bigsqcup_{j=1}^{m} G_{j}\right) * K_{r}\right) & =\max \left\{0, \check{\mu}\left(\bigsqcup_{j=1}^{m} G_{j}\right)-r\right\} \\
& =\max \left\{0, \sum_{j=1}^{m} \check{\mu}\left(G_{j}\right)+\sum_{j=1}^{m} i_{H}\left(G_{j}\right)-r\right\}
\end{aligned}
$$


The following lemma will be useful in the next section. To express it succintly we introduce the following Boolean condition. For a graph $G$ and vertex $v \in G, T(v, G)$ is true if and only if $v$ is a terminal vertex in some minimal path covering of $G$.

Lemma 7. Let $v \in G$ and $w \in H$.

$$
\mu((G \sqcup H)+v w)= \begin{cases}\mu(G \sqcup H)-1 & \text { if } T(v, G) \text { and } T(w, H) \\ \mu(G \sqcup H) & \text { otherwise }\end{cases}
$$

Proof. Let $\mu(G)=c, \mu(H)=d$ and $\mu((G \sqcup H)+v w)=t$. Clearly, $t \leqslant c+d$.

Let $R_{1}, \ldots, R_{t}$ be a minimal path cover of $(G \sqcup H)+v w$. If no $R_{i}$ contains $v w$ then this is also a minimal path cover of $(G \sqcup H)$ so $t=c+d$. Suppose $R_{1}$ contains $v w$ and note that $R_{1}$ is the only path with vertices in both $G$ and $H$. Removing $v w$ gives two paths $P \subseteq G$ and $Q \subseteq H$. Paths $P$ and $Q$ along with $R_{2}, \ldots, R_{t}$ cover $G \sqcup H$, so $t+1 \geqslant c+d$. Thus, $t$ can either be $c+d$ or $c+d-1$.

If $t=c+d-1$, then we have the minimal $(t+1)$-path covering $P, Q, R_{2}, \ldots, R_{t}$ of $G \sqcup H$, as above. We note that $v$ must be a terminal point of $P$ and $w$ must be a terminal point of $Q$, by construction. This path covering may be partitioned into a $c$-path covering of $G$ containing $P$ and a $d$-path covering of $H$ containing $Q$. Thus, $T(v, G)$ and $T(w, G)$ hold.

Conversely, suppose $T(u, G)$ and $T(w, H)$ both hold. Let $P_{1}, \ldots, P_{c}$ be a minimal path of $G$ with $v$ a terminal vertex of $P_{1}$ and let $Q_{1}, \ldots, Q_{d}$ be a minimal path cover of $H$ with $w$ a terminal vertex of $Q_{1}$. The edge $v w$ knits $P_{1}$ and $Q_{1}$ into a single path and $P_{1} \sim Q_{1}, P_{1}, \ldots, P_{c}, Q_{1}, \ldots, Q_{d}$ is a $c+d-1$ cover of $(G \sqcup H)+v w$. Consequently, $t \leqslant c+d-1$.

Thus, $T(u, G)$ and $T(w, H)$ both hold if and only if $t=c+d-1$. Otherwise, $t=c+d$.

Corollary 8. Let $v \in G$ and $w \in H$.

$$
\check{\mu}((G \sqcup H)+v w)= \begin{cases}\check{\mu}(G \sqcup H)-2 & \text { if } G=H=K_{1} \\ \check{\mu}(G \sqcup H)-1 & \text { if } T(v, G) \text { and } T(w, H) \\ \check{\mu}(G \sqcup H) & \text { Otherwise }\end{cases}
$$

Proof. Let $\delta=1$ if $T(v, G)$ and $T(w, H)$ are both true and $\delta=0$ otherwise. Then

$$
\begin{aligned}
\check{\mu}((G \sqcup H)+v w) & =\mu((G \sqcup H)+v w)-i_{H}((G \sqcup H)+v w) \\
& =\mu\left((G \sqcup H)-\delta-i_{H}((G \sqcup H)+v w)\right.
\end{aligned}
$$

The final term is -1 if and only if $G=H=K_{1}$.

\section{Decomposing Maximal $t$-path traceable graphs}

In this section we prove our main result, a maximal $t$-path traceable graph may be uniquely written as the join of a complete graph and a disjoint union of graphs that are also maximal 
with respect to traceability, but which are also either complete or have no universal vertex. We work with the families of graphs $\mathscr{M}_{t}$ for $t \geqslant 0$ and $\mathscr{N}_{t}$ for $t \geqslant 1$.

$$
\begin{aligned}
\mathscr{M}_{t} & :=\{G \mid \check{\mu}(G)=t \text { and } \check{\mu}(G+e)<t, \forall e \in E(\bar{G})\} \\
\mathscr{N}_{t} & :=\left\{G \in \mathscr{M}_{t} \mid G \text { is connected and has no universal vertex }\right\}
\end{aligned}
$$

The set $\mathscr{M}_{0}$ is the set of complete graphs. The set $\mathscr{M}_{1}$ is the set of graphs with a Hamiltonian path but no Hamiltonian cycle, that is, maximal non-Hamiltonian graphs. For $t>1, \mathscr{M}_{t}$ is also the set of graphs $G$ such $\mu(G)=t$ and $\mu(G+e)=t-1$ for any $e \in E(\bar{G})$. We will call these maximal t-path traceable graphs. A graph in $\mathscr{N}_{t}$ will be called trim.

Proposition 9. For $0 \leqslant s<t, G \in \mathscr{M}_{t}$ if and only if $K_{s} * G \in \mathscr{M}_{t-s}$.

Proof. We have $\check{\mu}\left(K_{s} * G\right)=\check{\mu}(G)-s$, so we just need to show that $K_{s} * G$ is maximal if and only if $G$ is maximal. The only edges that can be added to $K_{s} * G$ are those between vertices of $G$, that is, $E\left(\overline{K_{s} * G}\right)=E(\bar{G})$. For such an edge $e$,

$$
\begin{aligned}
\check{\mu}\left(\left(K_{s} * G\right)+e\right) & =\check{\mu}\left(K_{s} *(G+e)\right) \\
& =\check{\mu}(G+e)-s
\end{aligned}
$$

Consequently, $\check{\mu}(G+e)=\check{\mu}(G)-1$ if and only if $\check{\mu}\left(\left(K_{s} * G\right)+e\right)=\check{\mu}\left(K_{s} * G\right)-1$.

Note that the proposition is false for $s=t>0$ since $K_{s} * G$ will not be a complete graph and $\mathscr{M}_{0}$ is the set of complete graphs. The proof breaks down in (1).

Proposition 10. Let $G \in \mathscr{M}_{c}$ and $H \in \mathscr{M}_{d}$. The following are equivalent.

1. $G \sqcup H \in \mathscr{M}_{c+d+i_{H}(G)+i_{H}(H)}$

2. Each of $G$ and $H$ is either complete or has no universal vertex.

Proof. We have already shown that $\check{\mu}(G \sqcup H)=c+d+i_{H}(G)+i_{H}(H)$. We have to consider whether adding an edge to $G \sqcup H$ reduces the $\check{\mu}$-invariant. There are three cases to consider, the extra edge may be in $E(\bar{G})$ or $E(\bar{H})$ or it may join a vertex in $G$ to one in $H$. Since $G$ is maximal, adding an edge to $G$ is either impossible, when $G$ is complete, or it reduces the $\check{\mu}$-invariant of $G$. This edge would also reduce the $\breve{\mu}$-invariant of $G \sqcup H$ by Lemma 4. The case for adding an edge of $H$ is the same. Consider the edge $v w$ for $v \in V(G)$ and $w \in V(H)$. By Corollary 8 the $\check{\mu}$-invariant will drop if and only if $v$ is the terminal point of a path in a minimal path covering of $G$ and similarly for $w$ in $H$, that is, $T(v, G)$ and $T(w, H)$. Clearly this holds for all vertices in a complete graph. The following lemma shows that $T(v, G)$ holds for $G \in \mathscr{M}_{c}$ with $c>0$ if and only if $v$ is not a universal vertex in $G$. Thus, in order for $G \sqcup H$ to be maximal $G$ must either be complete, or be maximal itself, and have no universal vertex, and similarly for $H$.

As a key step before the main theorem, the next lemma shows that in a maximal graph, each vertex is universal, or a terminal vertex in a minimal path covering. 
Lemma 11. Let $c \geqslant 1$ and $G \in \mathscr{M}_{c}$. For any two non-adjacent vertices $v, w$ in $G$ there is a c-path covering of $G$ in which both $v$ and $w$ are terminal points of paths. Moreover, a vertex $v \in G$ is a terminal point in some c-path covering if and only if $v$ is not universal.

Proof. Suppose $c>1$ and let $v, w$ be non-adjacent in $G$. Since $G$ is maximal $G+v w$ has a $(c-1)$-path covering, $P_{1}, \ldots, P_{c-1}$. The edge $v w$ must be contained in some $P_{i}$ because $G$ has no $(c-1)$-path covering. Removing that edge gives a $c$-path covering of $G$ with $v$ and $w$ as terminal vertices. The special case $c=1$ is well known, adding the edge $v w$ gives a Hamiltonian cycle, and removing it leaves a path with endpoints $v$ and $w$. A consequence is that any non-universal vertex is the terminal point of some path in a $c$-path covering.

Suppose $P_{1}, \ldots, P_{c}$ is a $c$-path covering of $G \in \mathscr{M}_{c}$ with $v$ a terminal point of $P_{i}$. Then $v$ is not adjacent to any of the terminal points of $P_{j}$ for $j \neq i$, for otherwise two paths could be combined into a single one. In the case $c=1, v$ cannot be adjacent to the other terminal point of $P_{1}$, otherwise $G$ would have a Hamiltonian cycle. Consequently a universal vertex is not a terminal point in a $c$-path covering of $G$.

Theorem 12. For any $G \in \mathscr{M}_{t}, t>0, G$ may be uniquely decomposed as $K_{s} *\left(G_{1} \sqcup \ldots \sqcup G_{r}\right)$, where $s$ is the number of universal vertices of $G$, and each $G_{j}$ is either complete or $G_{j} \in \mathscr{N}_{t_{j}}$ for some $t_{j}>0$. Furthermore $t=\sum_{j=1}^{r} t_{j}+\sum_{j=1}^{r} i_{H}\left(G_{j}\right)-s$.

Proof. Suppose $G \in \mathscr{M}_{t}$ and let $s$ be the number of universal vertices of $G$. Let $r$ be the number of components in the graph obtained by removing the universal vertices from $G$, let $G_{1}, \ldots G_{r}$ be the components and let $\check{\mu}\left(G_{j}\right)=t_{j}$.

Proposition 6 shows that $t=\sum_{j=1}^{r} t_{j}+\sum_{j=1}^{r} i_{H}\left(G_{j}\right)-r$. By Proposition 9 , we have that $G \in \mathscr{M}_{t}$ if and only if $G_{1} \sqcup \ldots \sqcup G_{r} \in \mathscr{M}_{t+s}$. Furthermore, each $G_{j}$ must be in $\mathscr{M}_{t_{j}}$ for otherwise we could . Without loss of generality if we add an edge $e$ to $G_{1}$, such that $\check{\mu}\left(G_{1}+e\right)<t_{1}$, then

$$
\begin{aligned}
\check{\mu}(G+e) & =\check{\mu}\left(G_{1}+e\right)+\sum_{j=2}^{r} t_{j}+\sum_{j=1}^{r} i_{H}\left(G_{j}\right)-s \\
& <\sum_{j=1}^{r} t_{j}+\sum_{j=1}^{r} i_{H}\left(G_{j}\right)-s \\
& =t
\end{aligned}
$$

Now, we apply Proposition 10, so then $G_{1} \sqcup \ldots \sqcup G_{r} \in \mathscr{M}_{t+s}$, where $t+s=\sum_{j=1}^{r} t_{j}+\sum_{j=1}^{r} i_{H}\left(G_{j}\right)$ if and only if $G_{j}$ is either trim or complete. In other words, $G_{j} \in \mathscr{N}_{t_{j}}$ for $t_{j}>0$ or $G_{j} \in \mathscr{M}_{0}$ for $t_{j}=0$. 


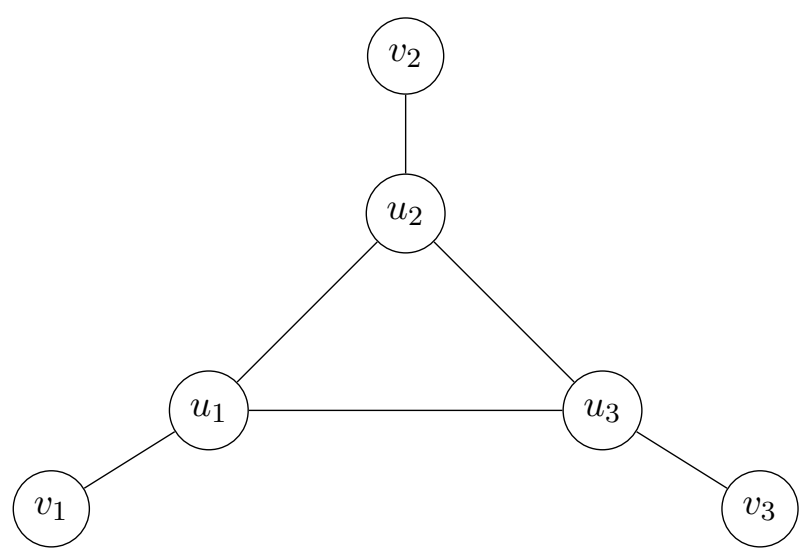

Figure 1: Smallest graph in $\mathscr{N}_{2}$

\section{Trim maximal $t$-path traceable graphs}

Skupien 6] discovered the first family of maximal non-Hamiltonian graphs, that is, graphs in $\mathscr{M}_{1}$. These graphs are formed by taking the join with $K_{r}$ of the disjoint union of $r+1$ complete graphs. The smallest graph in $\mathscr{N}_{2}$ is shown in Figure1. Chvátal identified its join with $K_{1}$ as the smallest maximal non-Hamilitonian graph that is not 1-tough, that is, not one of the Skupien family. Jamrozik, Kalinowski and Skupien [2] generalized this example to three different families. Family $A 1$ replaces each edge $u_{i} v_{i}$ with an arbitrary complete graph containing $u_{i}$ and replaces the $K_{3}$ formed by the $u_{i}$ with an arbitrary complete graph. The result has four cliques, the first three disjoint from each other but each intersecting the fourth clique in a single vertex. This graph is also in $\mathscr{N}_{2}$ and its join with $K_{1}$ gives a maximal non-Hamiltonian graph. Family $A 2$ is formed by taking the join with $K_{2}$ of the disjoint union of a complete graph and the graph in $\mathscr{N}_{2}$ just described. Theorem 12 shows that the resulting graph is in $\mathscr{M}_{1}$. Family $A 3$ is a modification of the $A 1$ family based on the graph in Figure 2, which is in $\mathscr{N}_{2}$. Bullock, Frick, Singleton and van Aardt [1] recognized that two constructions of Zelinka [7] gave maximal nontraceable graphs, that is, elements of $\mathscr{M}_{2}$. Zelinka's first construction is like the Skupien family: formed from $r+1$ complete graphs followed by the join with $K_{r-1}$. The Zelinka Type II family contains graphs in $\mathscr{N}_{2}$ that are a significant generalization of the graphs in Figures 1 and 2. In this section we generalize this family further to get graphs in $\mathscr{N}_{t}$ for arbitrary $t$. Our starting point is the graph in Figure 3, which is in $\mathscr{N}_{3}$.

Example 13. Consider $K_{m}$ with $m \geqslant 2 t-1$ and vertices $u_{1}, \ldots, u_{m}$. Let $G$ be the graph containing $K_{m}$ along with vertices $v_{1}, \ldots, v_{2 t-1}$ and edges $u_{i} v_{i}$. The case with $t=3$ and $m=5=2 t-1$ is Figure 3, We claim $G \in \mathscr{N}_{t}$.

One can readily check that this graph is $t$-path covered using $v_{2 i-1} \sim u_{2 i-1} \sim u_{2 i} \sim v_{2 i}$ for $i=1, \ldots, t-1$ and $v_{2 t-1} \sim u_{2 t-1} \sim u_{2 t} \sim \cdots \sim u_{m}$. We check that $G$ is maximal. By the symmetry of the graph, we need only consider the addition of the edge $v_{1} u_{m}$ and $v_{1} u_{2}$. In either case, the last and the first paths listed above may be combined into one, either

$$
\begin{aligned}
& v_{2 t-1} \sim u_{2 t-1} \sim \cdots \sim u_{m} \sim v_{1} \sim u_{1} \sim u_{2} \sim v_{2}, \quad \text { or } \\
& v_{2 t-1} \sim u_{2 t-1} \sim \cdots \sim u_{m} \sim u_{1} \sim v_{1} \sim u_{2} \sim v_{2}
\end{aligned}
$$




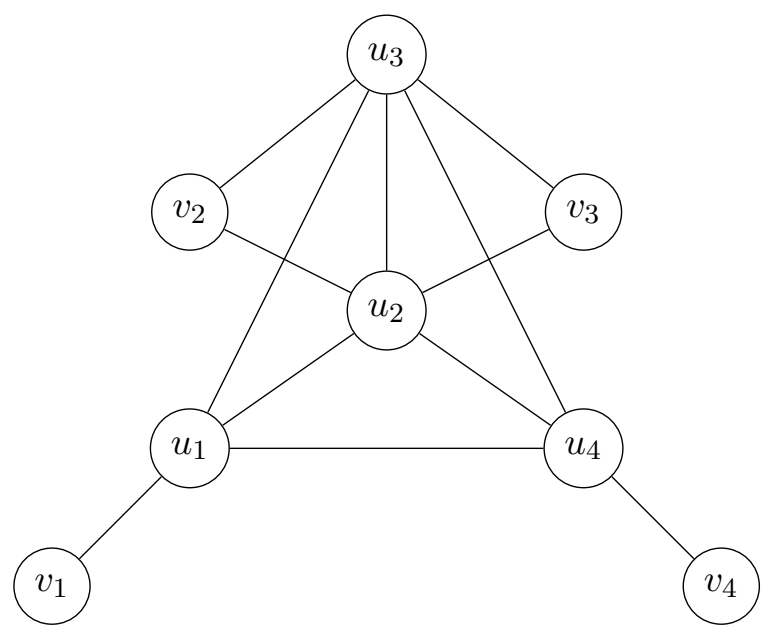

Figure 2: The join of this graph with $K_{1}$ is the smallest graph in the $A 3$ family.

Thus, adding an edge creates a $(t-1)$-path covered graph, proving maximality.

The next proposition shows that the previous example is the only way to have a trim maximal $t$-path covered graph with $2 t-1$ degree-one vertices. We start with a technical lemma.H

Lemma 14. Let $G$ be a connected graph and let $u_{1}, v_{1}, v_{2}, v_{3} \in G$ with $\operatorname{deg}\left(v_{i}\right)=1$, and $u$ adjacent to $v_{1}$ and $v_{2}$ but not $v_{3}$. Then $\mu(G)=\mu\left(G+u v_{3}\right)$.

Proof. Let $P_{1}, \ldots, P_{r}$ be a minimal path covering of $G+u v_{3}$; it is enough to show that there are $r$-paths covering $G$. If the covering doesn't include $u v_{3}$, then $P_{1}, \ldots, P_{r}$ also give a minimal path covering of $G$ establishing the claim of the lemma. Otherwise, suppose $u v_{3}$ is an edge of $P_{1}$. We consider two cases.

Suppose $P_{1}$ contains the edge $u v_{1}$ (or similarly $u v_{2}$ ). Then $P_{1}$ has $v_{1}$ as a terminal point and one of the other paths, say $P_{2}$ must be a length- 0 path containing simply $v_{2}$. Let $Q$ be obtained by removing $u v_{1}$ and $u v_{3}$ from $P_{1}$. Then $v_{1} \sim u \sim v_{2}, Q, P_{3}, \ldots, P_{r}$, gives an $r$-path covering of $G$

Suppose $P_{1}$ contains neither $u v_{1}$ nor $u v_{2}$. Then each of $v_{1}$ and $v_{2}$ must be on a length-0 path in the covering, say $P_{2}$ and $P_{3}$ are these paths. Furthermore $u$ must not be a terminal point of $P_{1}$, for, if were, the path could be extended to include $v_{1}$ or $v_{2}$, reducing the number of paths required to cover $G$. Removing $u$ from $P_{1}$ yields two paths, $Q_{1}, Q_{2}$. Then $v_{1} \sim u \sim v_{2}, Q_{1}, Q_{2}, P_{4}, \ldots, P_{r}$ gives an $r$-path cover of $G$. This proves the lemma.

Proposition 15. Let $G \in \mathscr{N}_{t}$. The number of degree-one vertices in $G$ is at most $2 t-1$. This occurs if and only if the $2 t-1$ vertices of degree-one have distinct neighbors and removing the degree-one vertices leaves a complete graph.

Proof. Each degree-one vertex must be a terminal point in a path covering. So any graph $G$ covered by $t$ paths can have at most $2 t$ degree-one vertices. Aside from the case $t=1$ and 


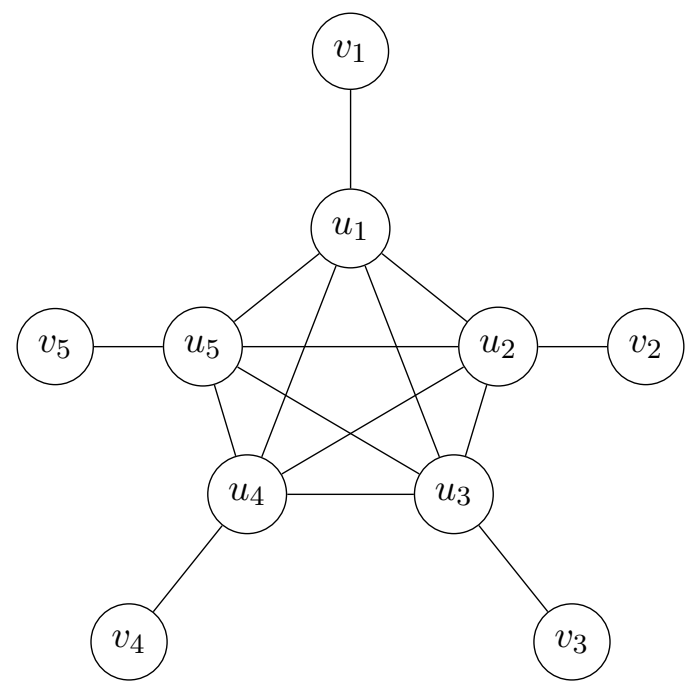

Figure 3: Whirligig in $\mathscr{N}_{3}$.

$G=K_{2}$, we can see that a graph with $2 t$ degree-one vertices cannot be maximal $t$-path traceable as follows. It is easy to check that a $2 t$ star is not $t$-path traceable (it is also not trim). A $t$-path traceable graph with $2 t$ degree-one vertices must therefore have an interior vertex $w$ that is not connected to one of the degree-one vertices $v$. Such a graph is not maximal because the edge $v w$ can be added leaving $2 t-1$ degree-one vertices. This graph cannot be $(t-1)$-path covered.

Suppose that $G \in \mathscr{N}_{t}$ with $2 t-1$ degree-one vertices, $v_{1}, \ldots, v_{2 t-1}$. Lemma 14 shows that no two of the $v_{i}$ can be adjacent to the same vertex, for that would violate maximality of $G$. So, the $v_{i}$ have distinct neighbors. Furthermore, all the nodes except the $v_{i}$ can be connected to each other and a path covering will still require at least $t$ paths since there remain $2 t-1$ degree-one vertices. This proves the necessity of the structure claimed in the proposition. The previous example showed that the graph is indeed in $\mathscr{N}_{t}$.

We can now generalize the Zelinka family.

Construction 16. Let $U_{0}, U_{1}, \cdots U_{2 t-1}$ be disjoint sets and $U=\bigsqcup_{i=0}^{2 t-1} U_{i}$. Let $m_{i}=\left|U_{i}\right|$ and assume that for $i>0$ the $U_{i}$ are non-empty, so $m_{i}>0$. For $i=1, \ldots, 2 t-1$ (but not $i=0$ ) and $j=1, \ldots, m_{i}$, let $V_{i j}$ be disjoint from each other and from $U$. Form the graph with vertex set $U \sqcup\left(\bigsqcup_{i=1}^{2 t-1}\left(\bigsqcup_{j=1}^{m_{i}} V_{i j}\right)\right)$ and edges $u u^{\prime}$ for $u, u^{\prime} \in U$ and $u v$ for any $u \in U_{i}$ and $v \in V_{i j}$ with $i=1, \ldots, 2 t-1$ and $j=1, \ldots, m_{i}$. The cliques of this graph are $K_{U}$ and $K_{U_{i} \sqcup V_{i j}}$ for each $i=1, \ldots, 2 t-1$ and $j=1, \ldots, m_{i}$.

The graph in Figure 2 has $m_{0}=0, m_{1}=m_{2}=1$ and $m_{3}=2$, and the graph in Figure 4 indicates the general construction. 


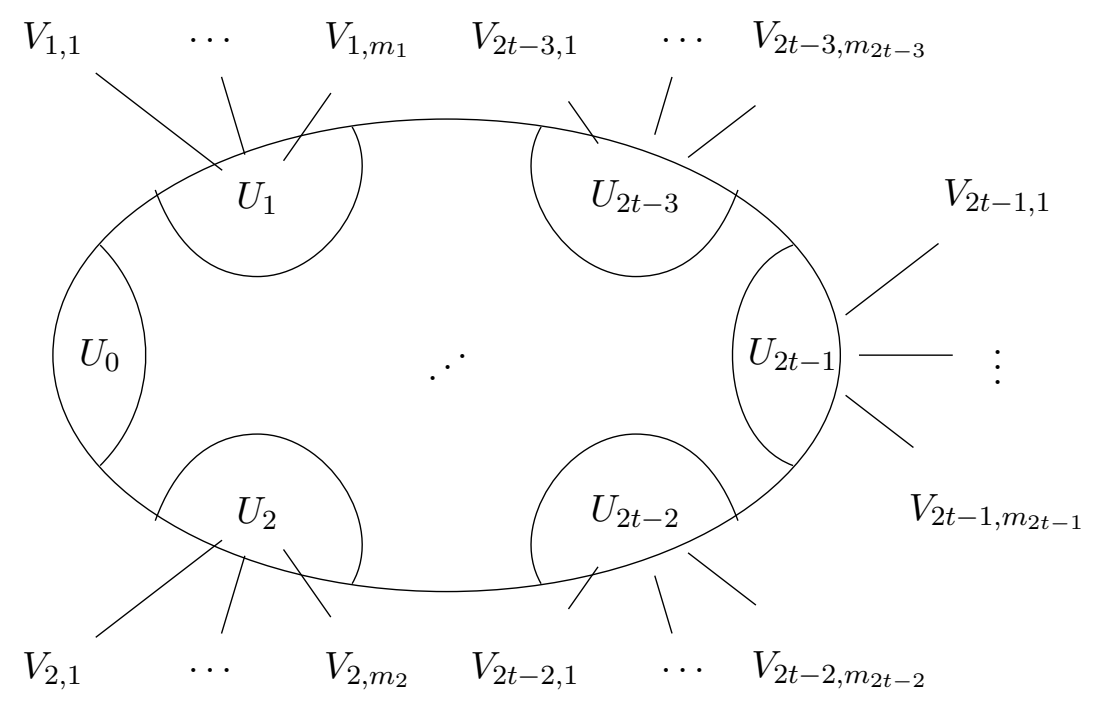

Figure 4: Generalization of the Whirligig, $W$

Theorem 17. The graph $W$ in Construction 16 is a trim, maximal t-path traceable graph.

Proof. We must show that $W$ is $t$-path covered and not $(t-1)$-path covered, and that the addition of any edge yields a $(t-1)$-path covered graph. The argument is analogous to the one in Example 13 .

Let $R$ be a Hamiltonian path in $U_{0}$. For each $i=1, \ldots, 2 t-1$ and $j=1, \ldots, m_{i}$ let $Q_{i j}$ be a Hamiltonian path in $K_{V_{i j}}$. Let $P_{i}$ be the path

$$
P_{i}: Q_{i 1} \sim u_{i 1} \sim \cdots \sim Q_{i m_{i}} \sim u_{i m_{i}}
$$

and let $\overleftarrow{P_{i}}$ be the reversal of $P_{i}$

Since there is an edge $u_{i m_{i}} u_{j m_{j}}$ there is a path $P_{i} \sim \overleftarrow{P}_{j}$ for any $i \neq j \in\{1, \ldots, 2 t-1\}$. Therefore the graph $W$ has a $t$-path covering $P_{2 i-1} \sim \overleftarrow{P}_{2 i}$ for $i=1, \ldots,(t-1)$, along with $P_{2 t-1} \sim R$. We leave to the reader the argument that there is no $(t-1)$-path cover.

To show $W$ is maximal we show that after adding an edge $e$, we can join two paths in the $t$-path cover above, with a bit of rearrangement. There are three types of edges to consider, the edge $e$ might join $V_{i j}$ to $U_{i^{\prime}}$ for $i \neq i^{\prime}$; or $V_{i j}$ to $V_{i j^{\prime}}$ for $j \neq j^{\prime}$; or $V_{i j}$ to $V_{i^{\prime} j^{\prime}}$ for $i \neq i^{\prime}$. Because of the symmetry of $W$, we may assume $i=1$ and $j=1$ and that the vertex chosen from $V_{i j}$ is the initial vertex of $Q_{i j}$. Other simplifications due to symmetry will be evident in what follows.

In the first case there are two subcases - determined by $i^{\prime} \geqslant 2 t$ or not - and after permutation, we may consider the edge $e$ from the initial vertex of $Q_{11}$ to the terminal vertex of $R$, or to the terminal vertex of $P_{2 t-1}$. We can then join two paths in the $t$-path cover: either $P_{2 t-1} \sim R \stackrel{e}{\sim}$ $P_{1} \sim \overleftarrow{P}_{2}$ or $P_{2} \sim \overleftarrow{P}_{1} \stackrel{e}{\sim} P_{2 t-1} \sim R$ 
Suppose next that we join the initial vertex of $Q_{11}$ with the terminal vertex of $Q_{12}$. We then rearrange $P_{1}$ and join two path in the $t$-path cover to get

$$
P_{2 t-1} \sim R \sim u_{11} \sim Q_{11} \stackrel{e}{\sim} Q_{12} \sim u_{12} \sim \cdots \sim Q_{1 m_{1}} \sim u_{1 m_{1}} \sim \overleftarrow{P}_{2}
$$

Finally, suppose that we join the initial vertex of $Q_{11}$ with the initial vertex of $Q_{2 t-1,1}$. Then we rearrange to $\overleftarrow{R} \sim \overleftarrow{P}_{2 t-1} \stackrel{e}{\sim} P_{1} \sim \overleftarrow{P}_{2}$

\section{References}

[1] F. Bullock, M. Frick, J. Singleton, S. van Aardt, K. Mynhardt, Maximal Nontraceable Graphs with Toughness less than One, Electronic Journal of Combinatorics 18 (2008), \#R18.

[2] J. Jamrozik, R. Kalinowski, Z. Skupien, A Catalogue of Small Maximal Nonhamiltonian Graphs, Discrete Mathematics39 (1982), 229-234.

[3] A. Marcyzk, Z. Skupien, Maximum nonhamiltonian tough graphs, Discrete Mathematics 96 (1991), 213-220.

[4] S. Noorvash, Covering the vertices of a graph by vertex-disjoint paths, Pacific Journal of Mathematics 58 (1975), 159-168.

[5] O. Ore, Arc Coverings of graphs, Ann. Mat. Ser. IV 55 (1961), 315-321.

[6] Z. Skupien, On Maximum non-Hamiltonian graphs, Rostock. Math. Kolloq. 11 (1979), 97-106.

[7] B. Zelinka, Graphs maximal with respect to absence of Hamiltonian Paths, Discussiones Mathematicae, Graph Theory 18 (1998), 205-208. 